\title{
Croiser l'évaluation de compétences didactiques et numériques sur un même support \\ Un défi à relever dans un enregistrement vidéo (curriQvidéo) en Master Enseignement Éducation et Formation du premier degré
}

Combining the assessment of didactic and digital skills on the same medium: A challenge to be taken up in video recording (curriQvidéo) in Master Teaching Education and Training of the first degree

Combinar la evaluación de habilidades didácticas y digitales en un mismo medio: un desafío a abordar en el grabación de vídeo (curriQvidéo) en Máster Docente en Educación y Formación de primer grado

https://doi.org/10.52358/mm.vi9.242

Christine Faller, enseignante agrégée, docteure en histoire Laboratoire LINE (UCA)

Enseignante à l'Institut supérieur du professorat et de l'éducation (INSPÉ) de Nice Université Côte d'Azur, France

christine.faller@univ-cotedazur.fr

Laurent Heiser, enseignant et docteur en sciences de l'information et de la communication. Laboratoire LINE (UCA) et IMSIC Toulon (UTLN)

Enseignant à l'Institut supérieur du professorat et de l'éducation (INSPÉ) de Nice

Université Côte d'Azur, France

laurent.heiser@univ-cotedazur.fr 
RÉSUMÉ

Cet article porte sur une recherche-action menée auprès d'étudiants professeurs du Master enseignement éducation et formation (MEEF) de l'INSPÉ de l'Université Côte d'Azur, parcours professeurs des écoles. II s'agit de leur faire produire une vidéo narrant le déroulé d'une séquence ou d'un projet pédagogique pour la classe dans laquelle ils sont en alternance (trois semaines en classe et trois semaines à l'INSPÉ). Cette production permet l'évaluation de deux modules de leur Master et semble, d'après les premiers résultats, constituer à la fois un objet de formation et un outil d'évaluation. La commande de leurs formateurs : s'inscrire dans une démarche de pédagogie active et placer leurs élèves en situation de technocréativité.

Mots-clés : évaluation, vidéo, scénarisation pédagogique, technocréativité, communication

\section{ABSTRACT}

This article focuses on action research carried out with student teachers of the master's in teaching education and training (MEEF) at the INSPÉ of Côte d'Azur University, school teacher paths. This involves having them produce a video narrating the progress of a sequence or an educational project for the class in which they are in work-study (three weeks in class and three weeks at INSPÉ). This production allows the evaluation of two modules of their master's degree and seems, according to the first results, to constitute both a training object and an evaluation tool. Instructors asked student teachersto be part of an active pedagogical approach and place their students in a techno-creativity situation.

Keywords: evaluation, vídeo, educational scripting, techno-creativity, communication

RESUMEN

Este artículo se centra en la investigación-acción llevada a cabo con estudiantes de profesorado del máster en docencia, educación y formación (MEEF) en el INSPÉ de Côte d'Azur University, cursos para profesores de escuela. Los estudiantes de profesorado deben producir un vídeo que narre el progreso de una secuencia o un proyecto educativo para la clase en la que se encuentran en el trabajo-estudio (tres semanas en clase y tres semanas en INSPÉ). Esta producción permite la evaluación de dos módulos de su máster y parece, según los primeros resultados, constituir tanto un objeto formativo como una herramienta de evaluación. La consigna de sus formadores: formar parte de un enfoque pedagógico activo y colocar a sus alumnos en una situación tecnocreativa.

Palabras clave: evaluación, video, guiones educativos, tecnocreatividad, comunicación 


\section{Introduction}

Nous sommes enseignants à l'Institut supérieur du professorat et de l'éducation (ci-après INSPÉ), tous deux docteurs après avoir eu une expérience professionnelle longue avec des élèves du secondaire. En passant à l'enseignement supérieur, nous avons pris la responsabilité de modules de formation et d'initiation à la recherche nous conduisant au même constat que celui mis en évidence par Bryk quant à la faible porosité des travaux de la recherche parmi les praticiens de terrain $(2015,467-477)$.

L'étude proposée ici s'inscrit dans ce qui a été caractérisé de « recherche dans et avec l'école » (Bianco, 2018, 121-128) en ce que l'expérimentation implique des « [...] acteurs scolaires mettant en œuvre une collaboration effective et intensive entre l'école et les chercheurs ». Les enseignants sont en même temps des étudiants de Master et des professeurs stagiaires, mais également des participants à notre projet de recherche. De ce fait, ces derniers se trouvent en parfaite adhésion avec les objectifs de notre recherche (précisés par la suite).

La formation initiale des enseignants au sein des INSPÉ a connu des modifications de maquettes de formation depuis septembre 2020. Elles s'inscrivent dans la continuité de l'obligation d'obtenir un Master pour devenir enseignant et visent à renforcer la professionnalisation en accentuant le lien entre pratique, recherche et formation. L'objectif de favoriser la posture réflexive des néoprofesseurs est particulièrement mise de l'avant dans les nouvelles maquettes de formation. Dès lors, ce point nous permet de rejoindre l'appel à contribution de la , puisque les étudiants vont être amenés à développer leur réflexivité en matière de littératie de l'évaluation.

Dans le cadre de leur évaluation, il est généralement d'usage de demander aux étudiants de Master 2 de constituer des dossiers ou portfolios décrivant et analysant leurs enseignements dans différentes disciplines lors de leurs stages. Face au constat d'insuffisances et d'incertitudes quant à la réalisation effective des activités décrites dans les portfolios, notamment avec le développement des sites de ressources en ligne, nous avons décidé, en 2017, d'abandonner ces dossiers. Nous nous sommes appuyés sur ce que vous avions l'habitude d'observer avec intérêt lors des visites de nos tutorés dans leurs classes à savoir leurs élèves en situation d'apprentissage. Aussi à la place des deux dossiers remis dans chacun des modules, nous avons proposé à tous nos étudiants de réaliser un curriQvidéo (Heiser et Faller, 2018).

Notre dispositif de formation, le CurriQvidéo, est à la fois une nouvelle approche pédagogique au sein de l'INSPÉ et une production d'évaluation réalisée par les étudiants. Celle-ci concerne une capsule vidéo de 5 minutes qui leur permet de faire une enquête sur la démarche curriculaire de leur séquence en partant de sa conception, jusqu'à en montrer le déroulé et évoquer les travaux des élèves. Pour le différencier d'une vidéo classique, nous avons eu recours à un néologisme : le nom de curriQvidéo est ainsi composé de « Curri » pour « démarche curriculaire », le « $Q$ » pour « questionnement » et « vidéo » pour la nature du support (Faller, 2021).

Le curriQvidéo s'inscrit volontairement dans l'innovation souhaitée par la réforme de la formation des enseignants en France. II vise à faire produire, par les étudiants, des vidéos qui leur permettent de valider deux modules de formation : I'EMC (enseignement moral et civique)/géographie/histoire d'une part et celui de culture et compétences numériques (CCNum) d'autre part. Ainsi, notre recherche se nourrit-elle d'un corpus de vidéos constitué par des productions d'enseignants débutants lorsqu'ils expérimentent une pédagogie active soutenue par le numérique dans deux champs d'enseignement qui deviennent 
dépendants l'un de l'autre. L'originalité de cette démarche provient du fait que la littérature sur la combinaison de ces deux didactiques n'est pas abondante, un constat qui nous conduit à vouloir en décrire le potentiel.

Depuis 2017, le dispositif CurriQvidéo est constitué d'un jeu de données de 500 vidéos. II a fait l'objet de plusieurs publications qui ont déjà examiné son cadre théorique sans jamais se focaliser sur l'une de ces particularités : l'évaluation.

Nous allons maintenant rappeler quelques points importants de ce cadrage théorique.

\section{Rappel du cadrage théorique}

Notre étude s'inscrit dans une démarche interdisciplinaire (Hakkarainen, 2011). Nous convoquons trois types de références, tout d'abord celui de plusieurs didactiques (géographie et EMC) et une approche sociocritique du numérique en éducation (Collin et al., 2015).

Nous inscrivons notre réflexion autour de ce dispositif de formation et d'évaluation dans plusieurs courants de recherche des sciences de l'éducation et de la formation pour la partie didactique, des sciences de l'information et de la communication pour ce qui concerne la forme scolaire et les enjeux du numérique, mais également des sciences numériques pour la conception des activités en lien avec la pensée informatique. Notre regard multidisciplinaire nous permet d'aborder la formation des nouveaux enseignants à la pédagogie active comme susceptible d'entraîner des contradictions par rapport aux attentes de la forme scolaire. Le support vidéo que rendent les enseignants stagiaires à la fin de la formation permet de raconter leur expérimentation d'une pédagogie contemporaine (Charlot, 2020) en lien avec le développement de compétences du $21^{\mathrm{e}}$ siècle (comme la pensée critique ou la pensée informatique). II permet également aux enseignants débutants de verbaliser les contradictions que cela peut générer en termes d'évaluation et les encourager à envisager d'autres modalités pour les dépasser.

\section{En didactique de la géographie}

L'épistémologie de la géographie scolaire nous mène au constat que son enseignement n'a cessé d'évoluer, par exemple, par l'insertion de la problématisation des leçons de géographie (Philippot, 2012). Nous pouvons affirmer que les choix didactiques sont motivés par l'évolution des sociétés. Ces derniers ont trouvé une traduction dans les programmes de 2016, enrichis en 2020. L'importance est donnée à l'environnement proche des élèves (Filâtre, 2020), le concept central pour l'école élémentaire étant celui d'habiter, c'est-à-dire la prise en compte de cinq fonctions en lien avec les pratiques quotidiennes des élèves (se loger, circuler, consommer, se divertir et travailler) ${ }^{1}$. La géographie en France est plus que jamais l'étude de la spatialisation des phénomènes et, de ce fait, cette approche s'intéresse à la société en tant que groupe producteur d'espace (Clerc, 2020).

\footnotetext{
${ }^{1}$ Le concept « habiter » est inscrit dans les programmes disponibles sur le site institutionnel EDUSCOL, https://cache.media.eduscol.education.fr/file/Geographie/74/4/RA16 C3 HIGE GEO CM1 Th1 Decouvrir lieu ou i-habite 616744.pdf
} 
En raison des bouleversements de notre accès aux savoirs via l'Internet, la géographie scolaire se devait de repenser les savoirs utiles pour tous les citoyens (Kirchberg, 2000) et les études récentes portant sur la géographie scolaire préconisent une géographie qui doit fasciner les enfants, les enrôler, les mettre en situation d'investigation à la fois pour comprendre le monde d'aujourd'hui, mais également constituer une aide à la décision pour les citoyens de demain. Ainsi, « travailler avec les outils géonumériques serait aussi un moyen pour réenchanter les cours de géographie en utilisant des outils qui fascinent de nombreux jeunes, mais dont ils méconnaissent parfois certains usages et surtout les dangers potentiels lors d'une mauvaise utilisation » (Mérenne-Schoumaker, 2016, p. 5).

Le choix qui a été fait dans l'approche de l'enseignement de la didactique de la géographie pour les étudiants concernés dans le corpus étudié est celui d'un raisonnement qui s'articule dans un espace, multiscalaire et dynamique (Mérenne-Schoumaker, 2012).

En outre, la géographie porte une forte dimension civique et elle doit contribuer à l'émancipation sociale et culturelle des élèves, elle doit aider à se dégager des idées reçues et de la pensée dominante, mais aussi forger son esprit critique afin de résister à une possible instrumentalisation de la géographie à des fins nationalistes ou racistes (Massey, 2006).

\section{En didactique de l'enseignement moral et civique (EMC)}

L'éducation à la citoyenneté a revêtu de nombreuses réalités et appellations dans le système éducatif français. Successivement, et même après avoir disparu un temps dans les années 70 , cet enseignement a connu des noms divers comme instruction civique, éducation civique, éducation à la citoyenneté et, au lycée, un enseignement d'éducation civique, juridique et social (ECJS). En 2008, le mot « morale » a fait son entrée, mais sur un mode qui a pris en compte les évolutions de la société (Caudon, 2007). La question du choix entre morale et éthique a été posée, mais assez rapidement tranchée par le choix de la notion de morale. Ce n'est qu'en 2015 que la mention unique - EMC (enseignement moral et civique) - a pris sa place dans tous les niveaux de classe (du primaire au lycée). Le dossier de veille de l'IFÉ2 sur la citoyenneté à l'école paru en 2018 (Ravez, 2018) a mis en évidence les recherches qui ont confirmé l'intérêt de sortir d'une morale transmissive ou encore de se limiter à l'amélioration du climat de classe pour entrer dans la proposition d'en assurer une réalité qui s'engage vers la notion d'expérimentation des valeurs (Bouteville et Falaize, 2014). En effet, la démarche qui prévaut en EMC se rattache à « la tradition inaugurée par Dewey, ces pratiques pédagogiques sont sous-tendues par une conception expérientielle des apprentissages qui nécessitent un retour réflexif sur l'activité » (Ravez, 2018, p. 26). Enfin, il ne s'agit plus d'imposer les valeurs par une leçon transmissive et explicative, mais bien de les faire comprendre aux élèves, de les rendre désirables tout en les tournant vers l'action (Prairat, 2015). Faire entrer les élèves dans des démarches actionnelles socialement responsables apparaissent comme particulièrement efficaces en matière de formation du citoyen (Geboers et al., 2015).

\footnotetext{
2 IFÉ : Institut français de l'éducation, intégré en 2011 à l'École normale supérieure de Lyon, dont la caractéristique est d'associer étroitement recherche et formation dans le domaine de l'éducation. Régulièrement, des dossiers de veille sont proposés et constituent à la fois des productions de synthèses rigoureuses, mais également des ressources mises à la disposition des chercheurs et des enseignants.
} 


\section{En didactique de la culture et compétences numériques}

La formation en culture et compétences numériques (CCNum) se situe au carrefour de plusieurs recherches et revendique son inscription dans l'approche sociocritique du numérique en éducation (Collin et al., 2015). Deux dimensions sont prises en compte ici. La première, systémique, permet de tenir compte du contexte socioculturel dans lequel les enseignants et les apprenants utilisent le numérique. Une seconde, dite critique, permet de réfléchir aux retombées d'une pédagogie soutenue par le numérique éducatif non seulement scolaires, mais aussi sociales et éducatives.

Dans ce contexte, la formation des enseignants au numérique prend appui sur l'idée que la technique fait partie du social, mais est également socialement inscrite dans nos pratiques. En ce sens, la pédagogie doit viser à enrichir le rapport de l'homme aux cybertechniques (Charlot, 2020), ce qui nécessite, pour l'enseignant, de concevoir des activités qui vont enrichir la citoyenneté des élèves à l'ère du numérique. Ces derniers vont donc devoir développer une connaissance minimum du numérique sans pour autant avoir à assimiler des connaissances techniques trop complexes.

Cette réflexion nécessite de s'appuyer sur des modèles bien connus d'intégration des technologies éducatives, comme le modèle SAMR (figure 1).

\section{Figure 1}

Modèle SAMR développé par Ruben Puentedura ${ }^{3}$

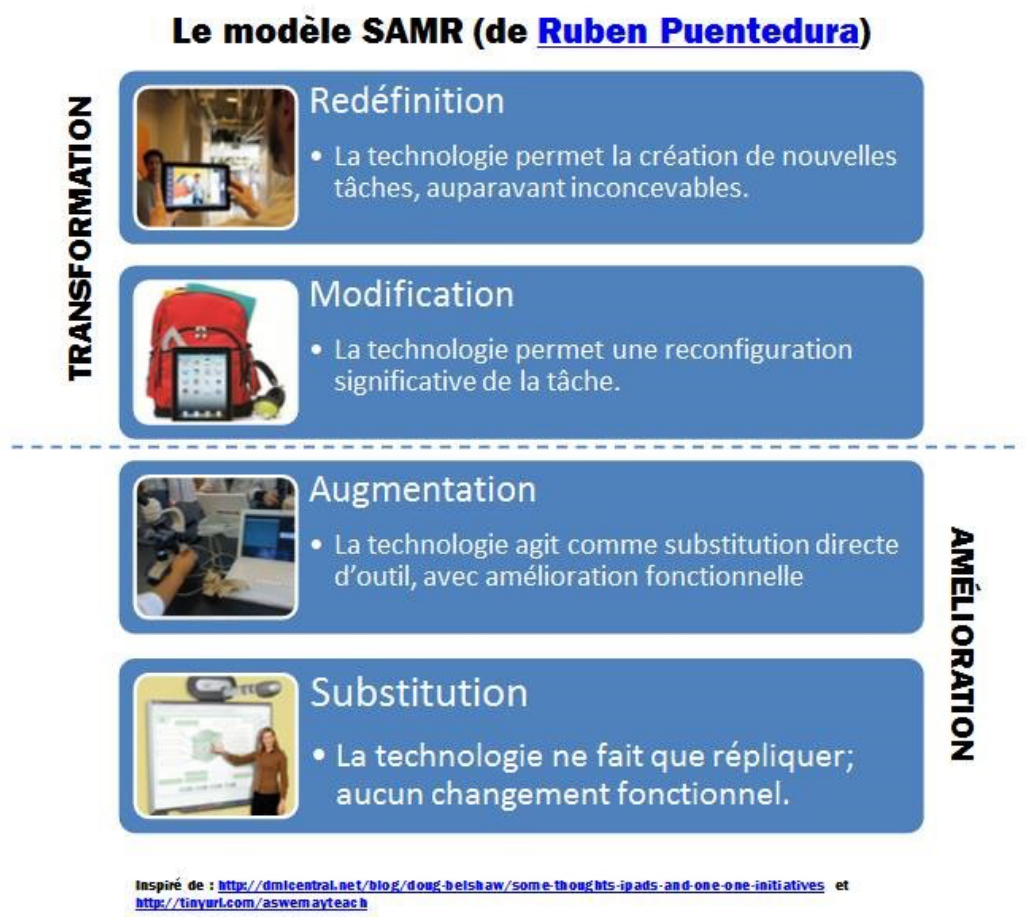

${ }^{3}$ Consulté le 6 février 2022 à : https://primabord.eduscol.education.fr/qu-est-ce-que-le-modele-samr 
Pour paraphraser le niveau supérieur du modèle, le numérique va permettre d'innover dès lors que les élèves vont réaliser des tâches scolaires comme il n'était pas possible de le faire avant. L'enseignant doit donc faire évoluer sa manière d'enseigner, ce qui peut influencer plusieurs critères comme l'usage du matériel, l'aménagement de la classe et l'évaluation. Ce modèle entre en résonance avec les conclusions de nombreux autres chercheurs ayant souligné que le numérique peut ne rien modifier à la pédagogie, voire même provoquer de la régression (Cerisier, 2016), ou encore que celui-ci est parfois intégré uniquement pour satisfaire les convenances de l'enseignant (Marquet, 2003).

Ainsi, pour atteindre l'innovation, l'enseignant ou le formateur doit tenir compte des niveaux d'usages du numérique en éducation, comme le propose, par exemple, le modèle passif participatif de Romero et al. (2017), présenté à la figure 2 .

\section{Figure 2}

Modèle passif participatif développé par Romero et al. (2017)

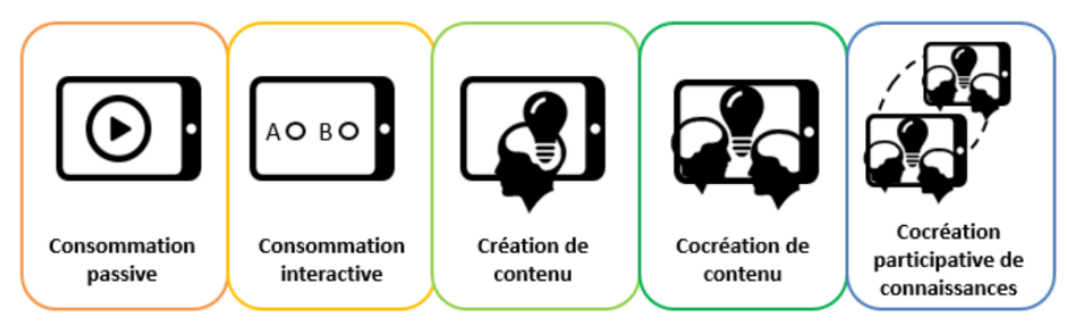

Le dernier niveau de ce modèle est à envisager dans la perspective d'une acculturation numérique des élèves. La pédagogie vise non seulement à enrichir leur rapport aux technologies, en leur permettant de mieux les comprendre, mais surtout à leur donner envie de s'en emparer pour devenir des citoyens actifs au sein de notre humanité numérique. Ou, dit autrement, la pédagogie des enseignants va chercher à les engager dans des tâches qui vont leur permettre d'enrichir leur maîtrise, leur connaissance et développer une attitude critique par rapport aux avancées technologiques. Pour que les futurs enseignants s'imprègnent des cinq niveaux d'usage du numérique en éducation, nous alimentons leur réflexion de référentiels de l'éducation nationale permettant de leur proposer une liste de toutes les compétences numériques. Le Cadre de référence des compétences numérique (CRCN), le Socle commun de connaissance et de culture, et des sites pédagogiques institutionnels servent cet objectif sans pour autant, selon nous, proposer d'éléments concrets sur l'évaluation. Cependant, nous misons sur le fait que la création des vidéos, devant servir à raconter l'expérimentation d'une pédagogie portée vers le développement des compétences numériques des élèves, va permettre d'envisager des stratégies d'évaluation conduisant à penser une littératie de l'évaluation qui dépasse l'évaluation par la note (donc sommative). 


\section{Problématique de l'article}

Nous venons de montrer que notre projet, en tant qu'outil de formation et d'évaluation, répond à des objectifs éducatifs quant à l'évolution du public élèves dont les enseignants ont en charge à l'heure des grandes mutations. Ainsi le curriQvidéo doit permettre de former des enseignants réflexifs pour qu'ils se tournent vers de nouvelles tâches scolaires (Heiser et al., 2020).

Après cinq ans d'expérimentation et dans l'état actuel de nos analyses, se posent 1) la question de la pertinence du curriQvidéo en matière d'évaluation d'une pédagogie innovante et 2) les limites pour y parvenir au sein de l'institution.

\section{La preuve de l'acquisition de compétences à la fois pour les enseignants et les élèves par le récit vidéo}

Le curriQvidéo va chercher à apporter la preuve de l'acquisition de compétences professionnelles en lien avec l'innovation, telles qu'elles se développent en classe à partir de situations concrètes d'enseignement et d'apprentissages. Tout au long de celui-ci, l'enseignant problématise et oriente ses formateurs pour prouver qu'il a acquis des compétences professionnelles simultanément dans les deux didactiques. Par exemple, le curriQvidéo peut poser la question : "Comment faire prendre conscience et engager les élèves dans un processus de lutte contre le harcèlement? ", ce qui peut donner lieu à une réalisation multimédia par les élèves, ou s'intéresser au recyclage en proposant un système de reconnaissance d'images ou un camion autonome capable de trier les déchets.

Enfin, l'enseignant veut montrer ses élèves en situation d'agir en les filmant directement comme acteurs des solutions proposées pour améliorer le vivre-ensemble.

Pour toutes ces raisons, il existe bien une cohérence dans le déroulé et la démarche curriculaire qui correspondent à la conception d'un scénario et son suivi dans sa classe.

Les critères qui vont permettre d'évaluer cette production finale sont :

- la problématique;

- l'acquisition de connaissances, de savoir-faire et de savoir-être;

- le climat créatif;

- les traces des apprenants (voir les élèves agir);

- la réflexivité de l'enseignant par rapport à ce qu'il donne à montrer (prise de recul sur son enseignement);

- la qualité globale du montage vidéo.

Chaque critère est ensuite évalué autour des quatre niveaux : absence, non acquis, en cours d'acquisition et acquis. L'évaluation, sous forme de grille, est instrumentée par la plateforme Moodle, ce qui permet d'illustrer chaque positionnement par des commentaires qui seront directement envoyés aux étudiants. Comme certains déposent la production finale avant l'échéance, il leur est accordé le droit de remanier certaines parties du montage vidéo. Dans les faits, peu d'étudiants utilisent cette opportunité, soit par manque de temps soit parce qu'il est leur est difficile de modifier le montage. Comme beaucoup de formateurs, notre évaluation par compétences doit être traduite sous forme de note selon les attentes 
institutionnelles. Les positionnements par niveaux, en cours d'acquisition ou acquis, sont traduits en une note qui leur permet d'atteindre facilement $15 / 20$ ou plus. L'essentiel, selon nous, demeure le niveau de compétence acquis pendant la formation et que le futur enseignant puisse se fixer des objectifs pour la suite de sa formation, après le Master, pendant les années de néotitulaire.

Il convient de préciser que les productions vidéo sont évaluées deux fois à l'aune des grilles de compétences spécifiques aux éléments constitutifs d'enseignement (ECUE), permettant à chacun des formateurs de conserver une certaine indépendance et une évaluation spécifique au regard des compétences de son module de formation. Notre évaluation, indépendante dans ses modalités, demeure soumise aux attentes de l'institution quant à la production de deux notes séparées. Le sens que nous donnons à notre travail n'est pas incompatible avec cette demande, mais elle nécessite des arts de faire (De Certeau, 1990) avec les limites que ce dernier peut produire au cours de l'expérience vécue du CurriQvidéo par les étudiants.

Tout au long de l'année, les étudiants sont accompagnés pendant les travaux dirigés et en dehors du temps universitaire par des échanges de mails. Les premiers cours sont consacrés à la méthodologie d'un curriQvidéo et à la construction des scénarios de séquences ou de projets pédagogiques. Puis durant l'année, chaque cours aborde les potentiels problèmes ou questions que se posent les étudiants. Enfin, à tout moment, les étudiants peuvent recevoir de l'aide individuelle lorsqu'ils rencontrent des difficultés. Ainsi, lors d'une expérience en classe de maternelle sur la problématique de la gestion de l'eau dans le jardin de l'école, l'installation faite avec les élèves dans le jardin ayant été vandalisée, nous avons accompagné l'enseignante stagiaire dans la réorientation de son projet avec ses élèves en transformant l'animation image par image ${ }^{4}$, ou stop motion, prévue sur la croissance des plantes en un stop motion sur le respect du travail d'autrui. Le curriQvidéo a été sauvé et les compétences, acquises.

Enfin, à l'issue du processus d'évaluation de la production d'une promotion, les étudiants professeurs ont été consultés dans le cadre de groupes de discussion (focus group) leur présentant trois questions : Qu'est-ce que le dispositif curriQvidéo vous a apporté? Quels ont été, pour vous, les limites et écueils de la réalisation d'un curriQvidéo? Quels conseils pourriez-vous donner aux formateurs? Les réponses des étudiants professeurs constituent des données utiles pour rendre la formation plus ciblée et plus efficiente, mais a aussi permis de mieux comprendre le ressenti des étudiants face à cette expérience et, par le fait même, celui de leurs élèves.

\section{La méthode de traitement des données}

Nous rappelons que cet article vise à analyser la pertinence du curriQvidéo et sa capacité à développer la réflexivité des enseignants débutants notamment en matière de littératie de l'évaluation.

Nous avons fait le choix d'appliquer une recherche à base de méthodes mixtes croisant l'analyse de données présentes dans les productions des étudiants et les données des groupes de discussion (Creswell et Plano Clark, 2011). La force de ce choix méthodologique réside dans le fait qu'elle croise des données qualitatives, qui proviennent des données réflexives sur l'expérience vécue, avec un bilan sur la conception d'une nouvelle pédagogie et la démarche d'évaluation que cela a nécessité. Durant notre étude, nous avons également pu mener des entretiens de groupe pour enrichir les résultats issus de l'analyse du corpus de curriQvidéos.

${ }^{4}$ https://gdt.oqlf.gouv.qc.ca/ficheOqlf.aspx?ld Fiche $=26540918$ 


\section{Le corpus de données}

Notre corpus est composé de plus de 500 curriQvidéos qui doit être appréhendé de façon chronologique, le dispositif ayant évolué au cours du temps. Nous avons fait le choix de traiter plus particulièrement les données des trois dernières années. Elles présentent à la fois des améliorations notables dans l'encadrement et l'accompagnement du dispositif, dans les équipements mis à la disposition des étudiants professeurs dans les écoles et elles nous donnent également des informations importantes sur les adaptations de la formation en temps de crise sanitaire (Romero, Heiser, Chiardola, Faller, 2020).

\section{POUR LES FORMATEURS : UNE ANALYSE À PARTIR DES ÉVALUATIONS PRÉSENTÉES DANS LES GRILLES}

Afin de répondre aux questionnements, nous avons décidé de dégager de grandes lignes directrices (Pasquier, 2018).

Pour répondre à nos questions de recherche, nous avons fait le choix d'analyser les CurriQvidéos selon trois axes :

- premièrement, en décrivant et en repérant des occurrences de choix pédagogiques, c'est-à-dire en cherchant à les catégoriser;

- deuxièmement, en analysant ces occurrences;

- troisièmement, en cherchant à repérer le terreau d'une pédagogie en devenir, les évolutions que cela peut entraîner sur le plan de leur rôle et de celui de leurs élèves comme celui d'un citoyen en devenir conscient des enjeux de société.

Précisons que nous ne chercherons pas à généraliser nos propos, mais à analyser ce qui, au sein des récits, permet de décrire des tensions à l'œuvre dans la forme scolaire. Comme nous venons de le dire, nous proposons une description de catégories puis une analyse de ces dernières et enfin une réflexion sur l'évolution de la forme scolaire. Ces trois niveaux doivent aboutir à comprendre comment l'EMC, ou la géographie, peut enrichir la culture du numérique.

Notre cadre méthodologique relève de l'interprétation des récits. Pour cela, nous avons élaboré une grille d'analyse à partir d'un algorithme de travail.

Cette stratégie a permis aux deux chercheurs de se fixer un travail par étapes (Paillé et Mucchielli, 2012). Tout d'abord, produire une description spontanée, puis tenter de raisonner sur celle-ci. Dans une dernière étape, les deux chercheurs se donnent rendez-vous pour comparer et confronter leurs analyses selon un plan de travail régulier et fixé à l'avance.

L'algorithme s'organise autour de quatre dimensions qui sont traditionnellement utilisées pour définir la forme scolaire (Vincent et al., 2012) : le temps, l'espace, les rapports sociaux, les savoirs. Nous avons également ajouté la dimension « autres » pour ne pas restreindre le travail de catégorisation (tableau 1). 


\section{Tableau 1}

Grille d'analyse des curriQvidéos

\begin{tabular}{|c|c|c|c|c|}
\hline \multicolumn{5}{|c|}{ CurriQvidéos / date_thématique_PC1 } \\
\hline Rubriques & $\begin{array}{l}\text { Description } \\
\text { spontanée }\end{array}$ & $\begin{array}{l}\text { Présentation plus } \\
\text { détaillée }\end{array}$ & $\begin{array}{l}\text { Raisonnement } \\
\text { spontané }\end{array}$ & $\begin{array}{l}\text { Analyse du } \\
\text { raisonnement }\end{array}$ \\
\hline \multicolumn{5}{|l|}{ Temps } \\
\hline \multicolumn{5}{|l|}{ Espace } \\
\hline \multicolumn{5}{|l|}{$\begin{array}{l}\text { Rapports } \\
\text { sociaux }\end{array}$} \\
\hline \multicolumn{5}{|l|}{ Savoirs } \\
\hline Autres & & & & \\
\hline
\end{tabular}

Nous avons procédé par blocs de curriQvidéos (un bloc $=10$ curriQvidéos). Tout d'abord, nous avons prélevé dix vidéos dans un corpus composé des productions des trois dernières années en prenant le soin de varier les thèmes abordés par les enseignants dans leur classe. Chaque chercheur remplit d'abord ses grilles seul, puis confronte ses résultats avec ceux de son collègue. Les échanges ont permis d'élaborer des grilles de synthèse qui présentent des résultats à analyser (quelques exemples sont présentés aux tableaux 2 et 3 ).

Le processus décrit ci-dessus est reconduit autant de fois que nécessaire jusqu'à saturation, c'est-à-dire jusqu'à obtenir une confirmation des aspects récurrents. 


\section{Tableau 2}

Extrait du tableau « Le temps »

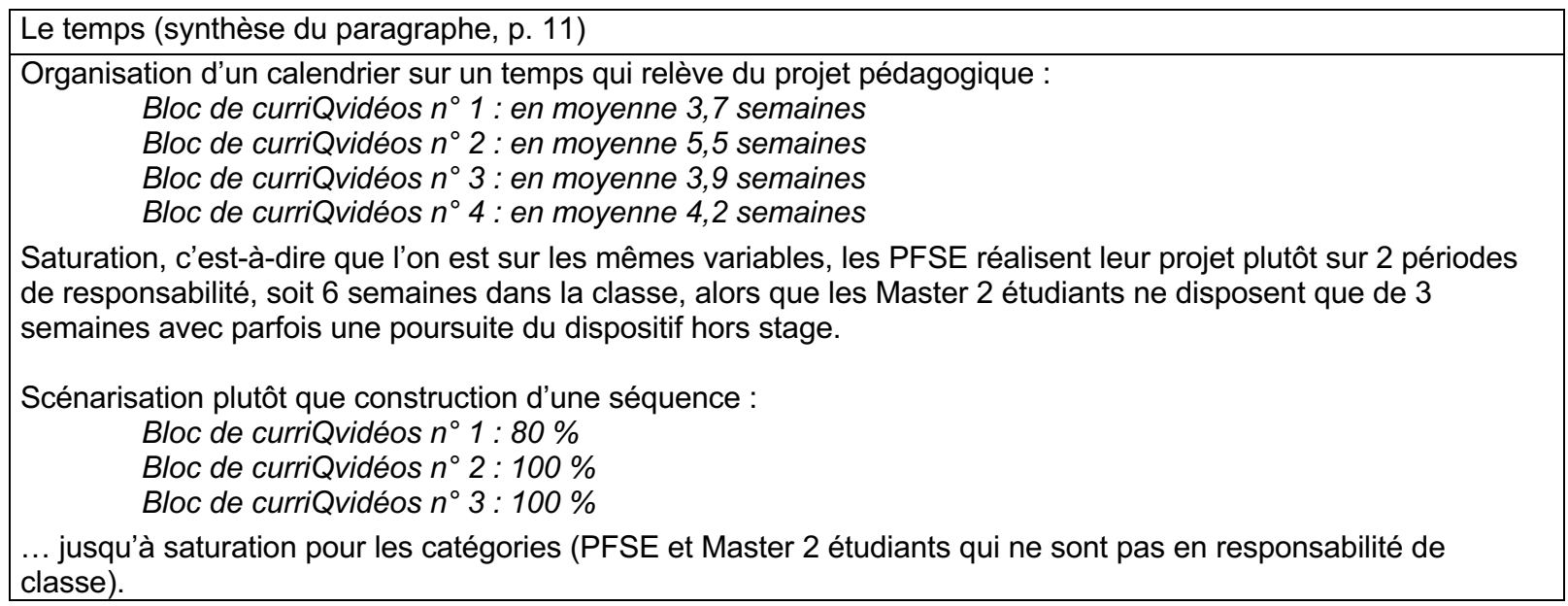

Note. II s'agissait dans cet élément de l'évaluation des items inscrits dans les critères des projets pédagogiques de Perrenoud, 2002.

\section{Tableau 3}

Extrait du tableau « L'espace»

L'espace : la synthèse du paragraphe, p. 11

Dans la vidéo, on voit les élèves qui ne sont pas seulement assis à leur place respective, mais ils se déplacent, ont une activité sur un tapis, une grille sur le sol...

Bloc de curriQvidéos $n^{\circ} 1: 100 \%$

Bloc de curriQvidéos $n^{\circ} 2: 100 \%$

Bloc de curriQvidéos $n^{\circ} 3: 100 \%$

Saturation, c'est-à-dire que l'on est sur les même constats (enseignants stagiaires, en responsabilité de classe et Master 2 étudiants en stages ponctuels).

Les élèves sortent de la classe (couloirs, cour de récréation, hors de l'enceinte de l'école, appréhendent des espaces plus larges comme le quartier, la ville jusqu'au monde).

Bloc de curriQvidéos $n^{\circ} 1$ : autrement qu'assis à sa place en classe : 100\% -Couloirs : $20 \%$ - salle motricité et cour : $50 \%$ - hors l'école : $20 \%$ )

Bloc de curriQvidéos $n^{\circ} 2$ : autrement qu'assis à sa place en classe :

100\% - Couloirs : $30 \%$ - salle motricité et cour : $40 \%$ - hors l'école : $20 \%)$

Bloc de curriQvidéos $n^{\circ} 3$ : autrement qu'assis à sa place en classe :

100\% - Couloirs : 10\% - salle motricité et cour : $60 \%$ - hors l'école : $40 \%)$

...jusqu'à saturation pour les catégories (PFSE et Master 2 étudiants qui ne sont pas en responsabilité de classe).

Note. Vérification du fait que la notion d'espace se construit dans une démarche inductive puis progresse dans la compréhension d'un emboîtement des échelles (du local au global). 


\section{Les résultats}

Voici les résultats de nos analyses à partir de la méthodologie présentée. Notre évaluation constitue un accompagnement engageant les étudiants professeurs sur la voie de la transformation à des degrés divers.

\section{Le temps}

Constat d'une forme de scénarisation dans le curriQvidéo sur un temps assez long au regard d'une année scolaire, généralement de trois à neuf semaines. Cela s'explique par le fait que les séquences présentées relèvent davantage du projet pédagogique pour les élèves. Ces derniers ont parfois aussi un rôle à jouer dans le temps de la séquence, car à plusieurs reprises, à la suite de l'engouement des élèves devenus force de proposition, le nombre de séances consacrées au projet s'allonge.

Quant au support de l'évaluation, le temps est aussi celui qui relève du tutoriel. On passe de la construction d'une séquence à celle d'un scénario, ce qui peut être expliqué par le support vidéo demandé aux étudiants professeurs. Une distinction est à noter entre les étudiants professeurs qui ne disposent pas de classe en responsabilité, mais qui sont accueillis par des maîtres d'accueil dans des classes durant quelques semaines (les Masters 2 EEF qui n'ont pas été lauréats du concours à l'issue du Master 1) et les professeurs fonctionnaires stagiaires qui sont lauréats du concours et en cours de titularisation en responsabilité d'une classe à l'année. Les différences sont notables, justement par le facteur temps. Les étudiants sans classe en responsabilité déplorent le manque de temps et racontent souvent ce qu'ils auraient pu faire, améliorer. Ils se projettent dans leur future classe, ce qui permet au curriQvidéo de rester pertinent, selon eux.

\section{L'espace}

Les curriQvidéos nous montrent qu'il existe une forme de déconstruction de l'espace classe au sens géographique du terme (Clerc, 2020). Mais dans la majeure partie des cas, ces sorties du cadre sont particulièrement adaptées à l'agir des élèves nécessaire dans le cadre de productions envisagées par les protagonistes du projet pédagogique. Les élèves appréhendent l'espace en mouvement et travaillent en emboîtement d'échelles par une première appréhension de l'espace proche puis, par les moyens de la géomatique, ils prennent conscience des espaces plus lointains et de leurs représentations. Ces aspects sont particulièrement travaillés en formation, dans les cours de Master ( $1^{\text {re }}$ et $2^{\mathrm{e}}$ année) et attendus dans l'évaluation.

Dans ce domaine de la représentation de l'espace, les élèves sont invités à imaginer, à créer. La créativité est au cœur : l'espace est une dimension fondamentale en emboîtement d'échelles avec la classe, l'école et hors l'école (dimension de présence dans l'espace de la ville, mais aussi dans des espaces en virtuel, sur des sites archéologiques, dans des musées, dans la rue par Google Maps ou en déplacement du groupe classe...). Dans cet espace, un corps bouge en dehors des périodes assis à sa table d'école. Les élèves sont montrés circulant dans leur classe, ils sont davantage debout, penchés en groupe sur un objet d'étude (sur des feuilles de différentes dimensions, sur des maquettes, des robots à manipuler, des tablettes ou ordinateurs ...). Une pédagogie qui passe par le corps et ses différentes postures. Un paradigme pragmatique à la Dewey (Foucart, 2013), mais avec l'expérience, associée à de l'explicite. Les élèves se déplacent dans la classe, dans les allées, restent debout pour 
filmer, discuter, coopérer; ces mouvements ne sont plus le privilège des enseignants (Clerc, 2019). À cela s'ajoutent toutes sortes de déplacements virtuels grâce aux systèmes d'information géographique.

\section{Les rapports sociaux}

Au départ, forte est la présence de l'enseignant qui lance le processus, interroge et ensuite favorise une cohésion de groupe. Ce qu'il est intéressant de constater, c'est l'implication des élèves à divers moments du processus; au début pour la problématisation, par exemple lors d'un problème qui s'impose à la classe dans leurs rapports sociaux (harcèlement, violences dans la cour...) ou sur des problèmes plus larges de société comme la pollution, ou comment mieux habiter dans son quartier ou dans sa ville. Les élèves proposent, débattent, discutent; l'enseignant accompagne, conseille, explique, enseigne (connaissances et compétences) et n'hésite pas à entrer dans des négociations, à laisser des situations de choix à des élèves. Dans ce critère, nous avons également observé le glissement d'une pédagogie, qui au départ se veut constructiviste, vers une pédagogie pragmatique. II ne suffit pas de voir l'expérience, mais dans le curriQvidéo, l'enseignant l'explicite.

\section{Les savoirs}

Les disciplines sont entremêlées et leurs frontières se fondent, l'interdisciplinarité est présente dans l'intégralité du corpus; il y a également une forme de glissement et de fusion des didactiques qui finissent par s'effacer derrière les objets produits. Tous les types de savoirs sont présents au service des compétences à acquérir dans les différents cycles de l'enseignement primaire. Autre glissement constaté : celui des ambitions didactiques à des savoirs contextualisés. Le curriQvidéo le montre à partir des images et vidéos présentes dans les travaux d'élèves en situation d'agir, et ces images et vidéos ont été sélectionnées par les étudiants professeurs pour le sens qu'ils leur donnent.

En prolongement de la réflexion et au regard de la situation actuelle de crise sanitaire, nous avons fait le constat que, sur les deux années scolaires impactées, les apprentissages et les questionnements opérés pour la conception des curriQvidéos a favorisé la mise en place d'une continuité pédagogique plus efficace qu'avec les méthodes traditionnelles que certains ont tenté de reproduire à distance. L'intérêt pour l'enseignant du $21^{\mathrm{e}}$ siècle est justement qu'il utilise le support vidéo pour construire des compétences; le support est le moteur au départ du projet, prétexte du recueil de données et, in fine, un constant aller-retour entre sa pratique et ce qui se passe dans la classe. Rien n'est complètement écrit dans ce scénario, mais l'enseignant est plutôt engagé dans une forme interactive entre lui et ses élèves.

Ces enseignants ne remettent pas en question le paradigme de l'école, mais ils le replacent dans ce que l'école annonce officiellement dans les discours sans vraiment y parvenir : questionner le monde avec leurs élèves ${ }^{5}$. Ils entrent véritablement dans la démarche de ce qu'est " éduquer » et notamment lorsqu'il s'agit de traiter des enjeux contemporains. Selon notre hypothèse, ils peuvent arriver à déployer cette pédagogie du fait d'être soutenus par l'INSPÉ, ce qui permet de lever ou de minimiser les contradictions vécues.

\footnotetext{
${ }^{5}$ Dans le cycle 2 de l'école élémentaire en France, les approches géographiques et historiques sont regroupées sous le libellé « Questionner le monde - espace et temps ».
} 


\section{Discussion finale et conclusions}

Nous avons précisé plus haut que nous souhaitions, par le CurriQvidéo, décrire et analyser les modalités pédagogiques telles qu'elles sont racontées par les enseignants stagiaires, mais aussi, dans le cadre de cet outil de formation et d'évaluation, repérer les évolutions afin de vérifier si ces modalités comportent :

1) Une mise en œuvre effective des pratiques didactiques et pédagogiques innovantes enseignées aux étudiants professeurs dans leurs formations même si cela est pondéré par des limites et des biais;

2) Des éléments de modification de leur représentation des usages du numérique dans les disciplines et du rôle des élèves dans des situations d'apprentissage;

3) Une plus-value aux enseignements de transmissions des connaissances et des cours de didactiques dispensés dans le cadre du Master au regard des nouveaux enjeux de notre société.

Nos résultats (voir exemples de nos tableaux de synthèse des recueils de données - tableaux 1 et 2 ainsi que les synthèses qui en ressortent à la section « Les résultats ») ont permis de présenter de grandes lignes directrices après des analyses et de nombreux échanges entre les deux formateurs impliqués et leurs étudiants.

Concernant les points 1 et 2 : bien plus qu'une énonciation de mise en œuvre dans les fiches de préparation de leurs cours, les étudiants enseignants montrent, en image et en son, des élèves en situation d'apprendre dans un projet où de nombreuses compétences sont nécessaires. Dans les situations montrées, les élèves se questionnent par la manipulation, par l'appropriation de l'espace, par des habiletés techniques construites pendant le temps scolaire grâce au scénario de l'enseignant (Faller, 2021).

Nous faisons également le constat que les élèves sont systématiquement mis en situation d'acteurs, modifiant souvent le cahier des charges établi par l'enseignant lors du lancement de la séquence et trouvent des solutions pour contourner les contraintes. Ainsi l'élève investigue, collabore à partir d'outils numériques comme les murs collaboratifs en ligne que leurs enseignants ont pu mettre à leur disposition. Dans ce renversement de pouvoir, l'enseignant n'est pas absent, il s'inscrit dans un accompagnement préconisé dans l'approche de John Dewey. Dans un même ordre d'idées, le curriQvidéo, par son objet et sa conception, place l'élève dans une dynamique que l'enseignant ne doit pas entraver : « son rôle est en effet d'accompagner les élèves pendant le travail coopératif, et de rester souple sur la structuration de ce travail » (Reverdy, 2016, p. 25). Ces élèves sont davantage en position d'ingénieurs que de chercheurs : ils échangent sur les moyens de rendre les processus opérationnels et ils sont également en mesure de détecter les obstacles majeurs à la réalisation de leur projet. Ils fixent, eux-mêmes, les limites du projet et ainsi le degré de créativité : Comment je peux représenter le système solaire dans un stop motion? ou encore Qu'est-ce qui va me permettre de prendre cette photo ou vidéo en incluant dans le champ de vision, tous les éléments que je veux voir dans mon film?

Notons qu'en période de crise sanitaire, cette pédagogie aura servi à lever certaines appréhensions, de la part des enseignants comme des élèves, pour arriver à créer. Cette pédagogie a déjà laissé des traces; la preuve en est que les habiletés et démarches disruptives entreprises juste avant le confinement ont permis la mise en place d'une continuité pédagogique plus efficiente fondée sur des usages du numérique pour l'apprentissage. 
Sur le point 3 de notre discussion, nous concevons que ces vidéos sont l'expression d'une déstabilisation, à comprendre comme l'expression d'une réflexivité qui se situe à la croisée entre un modèle pédagogique bien installé dans la forme scolaire, celui de l'enseignement collectif et simultané, et un autre plus contemporain, plus en adéquation avec les mutations sociétales dont les contours sont encore difficiles à saisir (Charlot, 2020).

II existe certains biais présents dans le dispositif du curriQvidéo. Le dispositif de formation met l'étudiant professeur dans une position de narrateur qui fait des choix dans un corpus de données qu'il a prélevées tout au long de son enquête. Sa narration a un sens didactique scénarisant l'ordre dans lequel il a conçu son projet. Ce biais est atténué par le fait que les élèves sont montrés en situation d'agir et de s'exprimer ainsi par la présentation des productions concrètes des élèves.

L'ambition de ce dispositif d'évaluation est de faire du curriQvidéo un outil de formation (Terrien, 2021) pour les futurs enseignants et de favoriser leur entrée dans une pédagogie de l'innovation. Cependant, nos grilles d'observation nous ont conduits à observer un certain statu quo avec les normes de la forme scolaire.

Ainsi, ces vidéos ne relèveraient-elles pas de l'utopie et nos données ne constitueraient-elles pas davantage une expérience dans la forme scolaire en probable mutation? C'est le film d'une vision de l'enseignement que des enseignants du $21^{\mathrm{e}}$ siècle souhaitent mettre en place. À ce stade de leur formation, rien ne permet de dire que ces enseignants arriveront à généraliser cette pratique ayant parfois suscité beaucoup de questionnements. De plus, certains vont céder aux pressions de l'institution qui leur demande, au final, de fournir une évaluation sommative. Nous avons fait le constat que, dans aucune production rendue par nos étudiants professeurs, il n'est pas question de notes ou de classement des élèves; ce sont vraiment les compétences qui sont mises en avant. Les élèves ne cherchent pas à dépasser les autres, mais à se dépasser. Les réalisations des uns et des autres sont mises en commun et présentées à d'autres élèves d'autres classes, car elles se veulent utiles.

\section{Éléments de conclusion}

Le dispositif mis en place est une première étape dans l'entrée de nos étudiants professeurs dans une approche raisonnée du numérique et dans des attitudes technocréatives accompagnant leurs élèves à les suivre. Les premières données issues du suivi de quelques cohortes de nos étudiants devenus néotitulaires ne nous permettent pas, à ce jour, d'affirmer que nous avons su les convaincre d'entrer dans des démarches pédagogiques innovantes dans leurs pratiques quotidiennes pour deux raisons. Tout d'abord, durant les premières années de leur carrière, ces néoprofesseurs sont très souvent affectés à des postes de remplaçants ou de mi/tiers-temps dans des écoles parfois très éloignées de chez eux. Ensuite, l'équipement restreint de certaines écoles primaires des communes du département du Var entrave et ralentit l'entrée des enseignants et de leurs élèves dans des processus technocréatifs. Nous manquons actuellement de recul pour constituer un recueil de données nous informant sur leurs futures postures lors de leurs affectations à un poste définitif. Nous espérons avoir au moins pu leur démontrer qu'il est possible pour eux de tenter de faire évoluer les pratiques au profit de la formation de citoyens engagés et soucieux de ce qui les entoure. 


\section{Liste de références}

Bianco, M. (2018). La réponse à des questions cruciales en éducation réside-t-elle dans un changement de paradigme? Éducation et didactique, 12-1, p. 121-128. Consulté le 30 novembre 2021. URL : https://doi.org/10.4000/educationdidactique.3111

Bourmeau, S. (2020, 12 août). Avoir raison avec John Dewey, épisode 3 [podcast]. France culture. https://www.franceculture.fr/emissions/avoir-raison-avec/avoir-raison-avec-john-dewey-35-john-dewey-pedagogueexperimental

Bryk, A. (2015). Accelerating how we learn to improve. 2014 AERA Distinguished Lecture. Educational Researcher, 44(9), 467-477. https://doi.org/10.3102\%2F0013189X15621543

Caudon, H. (2007). Oser à nouveau enseigner la morale à l'école, Hachette éducation.

Cerisier, J.-F. (2016). La forme scolaire à l'épreuve du numérique. Dans TiceMed, Centre de Recherche sur les Médiations (Metz) et milieux Laboratoire de recherche Information médias, médiations (Sophia Antipolis, Alpes-Maritimes) (dir.), Numérique et éducation : Dispositifs, jeux, enjeux, hors-jeux (PUN-éditions universitaires de Lorraine, p. 195-209).

Charlot, B. (2020). Éducation ou barbarie. Economica, Anthropos.

Clerc, P. (2019). Chapitre 4. La longue histoire de la géographie à l'école. Géographies : Épistémologie et histoire des savoirs sur l'espace (p. 32-37). Paris : Armand Colin.

Clerc, P. (2020). La salle de classe : Un objet géographique. Géocarrefour, 94(94/1), article 94. https://doi.org/10.4000/geocarrefour.14426

Collin, S., Guichon, N., et Ntebutse, J. G. (2015). Une approche sociocritique des usages numériques en éducation. Sciences et Technologies de l'Information et de la Communication pour l'Éducation et la Formation, 22. https://hal.archives-ouvertes.fr/hal-01218240

De Certeau, M. (1990). L'invention du quotidien 1. Arts de faire, Paris, Gallimard.

Faller, C. (2020, février). « Techno créativité » dans le dossier L'aventure de la géographie. Les Cahiers pédagogiques, 559.

Faller, C. (2021, mai). « Un ricochet de créativité » dans le dossier Créativité. Les Cahiers pédagogiques, 569.

Filâtre E. (2020). La place de l'espace proche dans l'évolution des programmes de géographie de l'école élémentaire française de 1977 à 2015, Cybergeo: European Journal of Geography. Épistémologie, Histoire de la Géographie, Didactique, document 950. https://doi.org/10.4000/cybergeo.35167

Bouteville , E. Falaize, B. (2014). 15 séquences éducation citoyenne et morale. RETZ.

Foucart, J. (2013). Pragmatisme et transaction. La perspective de John Dewey. Pensée plurielle, 33-34, 73-84. https://doi.org/10.3917/pp.033.0073

Geboers, E., Geijsel, F., Admiraal, W., Jorgensen T. et ten Dam, G. (2015). Citizenship development of adolescents during the lower grades of secondary education. Journal of Adolescence, 45, 89-97. https://doi.org/10.1016/j.adolescence.2015.08.017

Hakkarainen, P. (2011). Promoting Meaningful Learning through Video Production-Supported PBL. Interdisciplinary Journal of Problem-Based Learning, 5(1). Available at: https://doi.org/10.7771/1541-5015.1217

Heiser, L., Romero, M., De Smet, C., Faller, C. (2020). Conception d'activités technocréatives pour le développement d'une pédagogie créative. Formation et profession, 28(2), 51-59. https://doi.org/10.18162/fp.2020.547

Heiser L., Faller C. (2018). Les CurriQvideo : Une approche enactive de formation à la techno créativité à l'ESPE de Nice. Journée de la Recherche en Éducation (JRE 2018 Tahiti), May 2018, Tahiti, France. https://hal.archives-ouvertes.fr/hal-02290918

Kirchberg, G. (2000). Changes in Youth: No Changes in Teaching Geography? Aspects of a Neglected Problem in the Didactics of Geography. International Research in Geographical and Environmental Education, 9(1). https://doi.org/10.1080/10382040008667626 
Marquet, P. (2003). L'impact des TIC dans l'enseignement et la formation : mesures, modèles et méthodes; contribution à l'évolution du paradigme comparatiste des usages de l'informatique en pédagogie. Éducation. Université Louis Pasteur - Strasbourg I.

Massey, D. (2006). The Geographical Mind. Dans Secondary Geography Handbook. The Geographical Association, 46-51. https://www.dcuci.univr.it/documenti/Avviso/all/all042035.pdf

Mérenne-Schoumaker, B. (2012). Didactique de la Géographie. Organiser les apprentissages, De Boeck.

Mérenne-Schoumaker, B. (2016). Pour un renouveau de la didactique de la géographie. Cybergeo : European Journal of Geography. (Les 20 ans de Cybergeo). http://journals.openedition.org/cybergeo/27746

Paillé, P. et Mucchielli, A. (2012). L'analyse qualitative en sciences humaines et sociales. Armand Colin. https://doi.org/10.3917/arco.paill.2012.01

Pasquier D. (2018). L'internet des familles modestes : Enquête dans la France rurale. Nouvelle édition, Presse des mines, Paris.

Perrenoud, P. (2002). Apprendre à l'école à travers des projets : pourquoi? comment?, Université de Genève. http://www.unige.ch/fapse/SSE/teachers/perrenoud/php main/php 1999/1999 17.html

Philippot, T. (2012). Enseigner à l'école primaire une géographie problématisée : un défi? Nouveaux cahiers de la recherche en éducation, 15(1), 21-34. https://doi.org/10.7202/1013377ar

Prairat, E. (2015). Les valeurs. Des interrogations du philosophe au défi du pédagogue. Diversité, 182, 80-84.

Ravez, C. (2018). Regards sur la citoyenneté à l'école. Dossier de veille de l'IFÉ, (125), p. 26. ENS de Lyon. http://veille-et-analyses.ens-lyon.fr/DA/detailsDossier.php?parent=recherche\&dossier=125\&lang=fr

Reverdy, C. (2016). La coopération entre élèves : des recherches aux pratiques. Dossier de veille de l'IFÉ, (114), p. 25. ENS de Lyon. http://veille-et-analyses.ens-lyon.fr/DA/detailsDossier.php?parent=recherche\&dossier=114\&lang=fr

Romero, M., Lille, B., et Patino, A. (dir.). (2017). Usages créatifs du numérique pour l'apprentissage au XXI ${ }^{\mathrm{e}}$ siècle (vol. 1). Presses de l'Université du Québec.

Romero, M., Heiser, L., Chiardola, A., Faller, C. (2020). Poursuivre le programme ou assurer l'engagement? Analyse des critères de continuité pédagogique et des transformations pédagogiques et en contexte de pandémie. Formation et profession, 28 (4; hors-série), 1-15. https://doi.org/10.18162/fp.2020.692

Terrien, P. (2021). Modéliser les dispositifs de formations innovants? Deux exemples à l'étude. Université de Namur. $L a$ question de l'identité de la formation culturelle du corps enseignant, Presses universitaires de Namur, p. 39 (978-239029-130-5). https://hal-amu.archives-ouvertes.fr/hal-03214342

Vincent, G., Courtebras, B. et Reuter, Y. (2012). La forme scolaire : débats et mises au point : Entretien de Guy Vincent avec Bernard Courtebras et Yves Reuter. Recherches en didactiques, 1(1), $109-135$. https://doi.org/10.3917/rdid.013.0109 